\title{
A submillimetre search for pre- and proto-brown dwarfs in Chamaeleon II
}

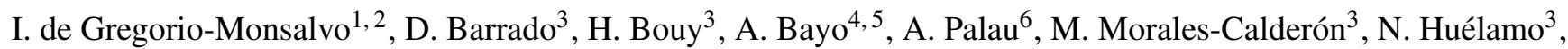 \\ O. Morata ${ }^{7}$, B. Merín ${ }^{8}$, and C. Eiroa ${ }^{9}$
}

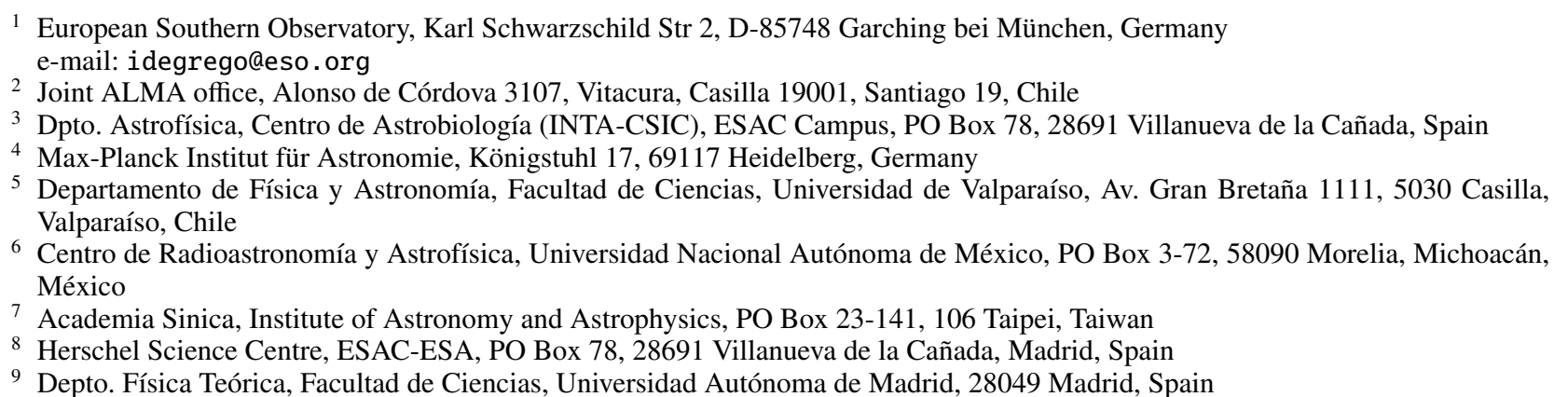

Received 6 May 2014 / Accepted 1 December 2015

\begin{abstract}
Context. The Chamaeleon II molecular cloud is an active star-forming region that offers an excellent opportunity to study the formation of brown dwarfs in the southern hemisphere.

Aims. Our aims are to identify a population of pre- and proto-brown dwarfs ( $5 \sigma$ mass limit threshold of $\sim 0.015 M_{\odot}$ ) and provide information on the formation mechanisms of substellar objects.

Methods. We performed high sensitivity observations at $870 \mu \mathrm{m}$ using the LABOCA bolometer at the APEX telescope towards an active star-forming region in Chamaeleon II. The data are complemented by an extensive multiwavelength catalogue of sources, which covers the optical to the far-infrared, to study the nature of the LABOCA detections.

Results. We detect 15 cores at $870 \mu \mathrm{m}$, and 11 of them show masses in the substellar regime. The most intense objects in the surveyed field correspond to the submillimetre counterparts of the well-known young stellar objects DK Cha and IRAS 12500-7658. We identify a possible proto-brown dwarf candidate (ChaII-APEX-L) with IRAC emission at 3.6 and $4.5 \mu \mathrm{m}$.

Conclusions. Our analysis indicates that most of the spatially resolved cores are transient, and that the point-like starless cores in the substellar regime (with masses between $0.016 M_{\odot}$ and $0.066 M_{\odot}$ ) could be pre-brown dwarfs cores that are gravitationally unstable if they have radii less than $220 \mathrm{AU}$ to $907 \mathrm{AU}\left(1.2^{\prime \prime}\right.$ to $5^{\prime \prime}$ at $178 \mathrm{pc}$ ), respectively, for different masses. ALMA observations will be key to revealing the energetic state of these pre-brown dwarfs candidates.
\end{abstract}

Key words. stars: pre-main sequence - stars: formation - stars: low-mass - brown dwarfs

\section{Introduction}

The formation of brown dwarfs (BDs) is still a hot topic of research. The most widely discussed scenarios for their formation include turbulent fragmentation (Padoan \& Nordlund 2004), ejection from multiple protostellar systems (Reipurth \& Clarke 2001; Bate et al. 2002; Bate 2012), disk fragmentation and subsequent ejection (Stamatellos \& Whitworth 2009), and photo-evaporation of massive pre-stellar cores (Whitworth $\&$ Zinnecker 2004). Since stars and brown dwarfs evolve very rapidly during the first million years, many answers to the formation mechanism (or mechanisms) have to come from the study of their properties when they are deeply embedded in the natal cloud, what we call the "proto-BD" stage (which would correspond to the Class 0/I stage in the classical evolutionary scheme of young stellar objects (YSOs); Lada 1987; André et al. 1993, 2000). If we find proto-BDs surrounded by substantial envelopes, similar to the ones observed in the first stages of lowmass protostars (André et al. 2000), they would provide direct support for the in situ formation of brown dwarfs. Thus, sensitive millimetre and submillimetre surveys offer the exciting prospect of identifying a population of proto-BD candidates for the first time.

Previous works aiming to study the earliest stages of the formation of BDs in the millimetre/submillimetre regime have followed two different approaches: core surveys (mapping the dust continuum emission in a region of a molecular cloud to detect the presence of substellar cores, which are limited by the signalto-noise ratio $(\mathrm{S} / \mathrm{N})$ achieved and the extension of the maps; e.g. Greaves et al. 2003), and the study of individual objects using previous information at other wavelengths (e.g. Barrado et al. 2009; André et al. 2012; Palau et al. 2012, 2014; Lee et al. 2013).

The development of a new generation of submillimetre bolometer arrays like $\mathrm{LABOCA}^{1}$ at the $\mathrm{APEX}^{2}$ telescope together with the excellent weather conditions of the site (Llano

\footnotetext{
http://www . apex-telescope.org/bolometer/laboca/

http://www . apex-telescope.org/
} 

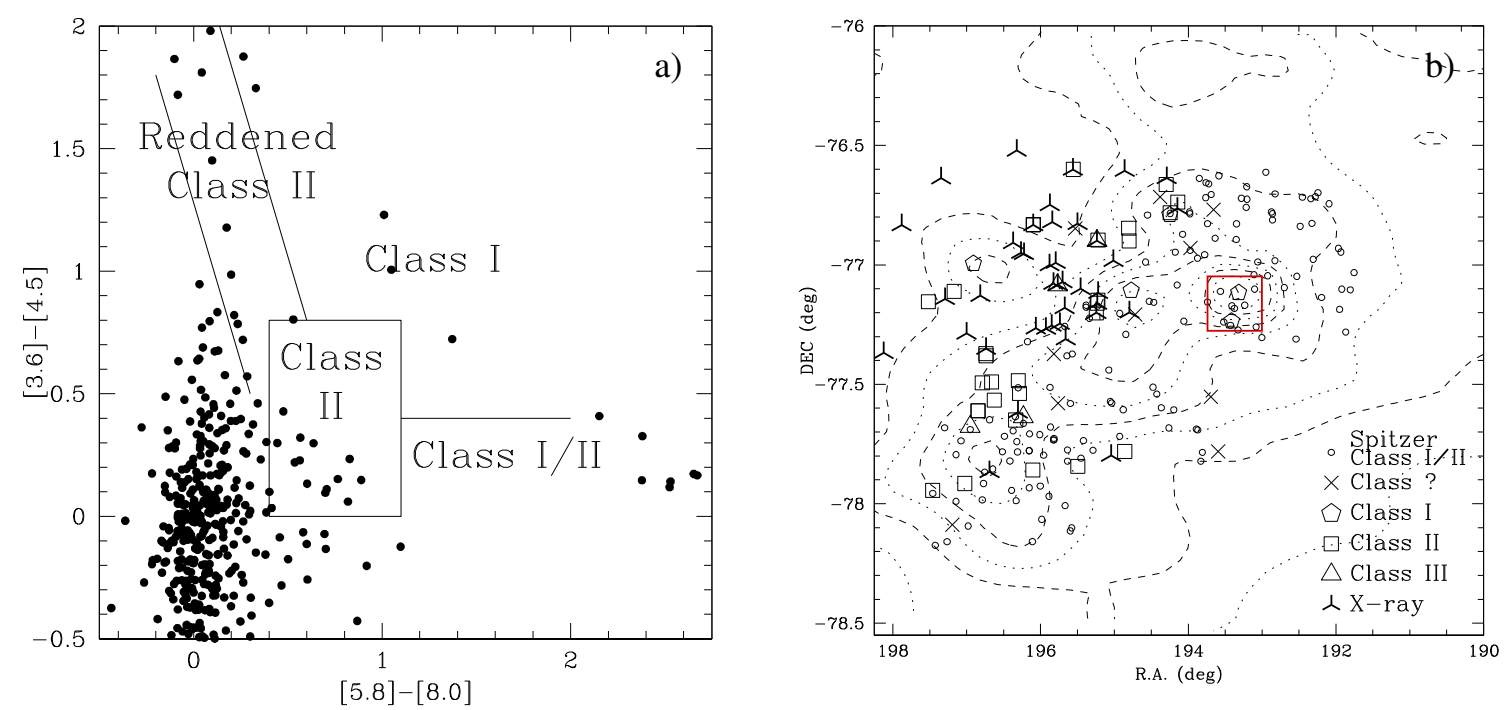

Fig. 1. a) Spitzer/IRAC colour-colour diagram, with a classification for Class I, II, and III for candidate members in Cha II. b) Distribution of possible members of Cha II on the sky. The contours correspond to an IRAS map at 100 microns. X-ray sources appear as three-point stars (mostly weak-line T Tauri stars), from the literature. New candidate members, based on our analysis of Spitzer data, are displayed as triangles, squares, pentagons, crosses, and small circles (sources with evolutionary stages between Class I and II), depending on the evolutionary class (i.e. the IRAC IR excess, from a colour-colour diagram following Allen et al. 2004). Red square shows the region selected to be observed with LABOCA, which includes two out of the three Class I objects of the Cha II cloud.

Chajnantor, $5000 \mathrm{~m}$ high), provide an excellent opportunity to carry out core surveys that map extended regions at high sensitivity in a reasonable amount of time.

The southern star-forming region in Chamaeleon consists of three major dark clouds - Chamaeleon I, II, and III - and a handful of smaller structures (Schwartz 1977). Given its relative proximity (160-180 pc), youth (1-3 Myr), and intermediate galactic latitude $(b \sim-17 \mathrm{deg})$, this cloud complex is well suited for investigations of low-mass stellar and substellar populations.

We are particularly interested in the Chamaeleon II (Cha II) region since it seems to be in the earliest stage of evolution, compared to the Cha I and Cha III regions, which offers an excellent opportunity to study the formation of brown dwarfs: a number of pre-main-sequence stars with $\mathrm{H} \alpha$ emission were identified in this region (Schwartz 1977; Hartigan 1993). Near-infrared and IRAS data confirmed that these sources likely harbour circumstellar material (e.g. Larson et al. 1998).

$\mathrm{An} \mathrm{ISO}^{3}$ study reveals four new candidate YSOs, including a very low luminosity $\left(\geq 0.01 L_{\odot}\right)$ source with mid-infrared excess (Persi et al. 2003). Alcalá et al. (2000) detect 40 X-ray sources using ROSAT ${ }^{4}$ observations (only 14 of these sources coincided with previously known YSOs) and suggest that Cha II contains a lower number of weak-line T Tauri stars (Class III) than classical T Tauri stars (Class II), unlike the rest of Chamaeleon major dark clouds (Cha I and III), which suggests that Cha II is the one in the earliest stage of evolution. In addition, Vuong et al. (2001) using $I, J, K_{\mathrm{S}}$ data from the DEep Near Infrared Survey of the southern sky (DENIS ${ }^{5}$; Epchtein et al. 1997) identified 51 candidate lowmass stars and brown dwarfs in the region. Comparison with theoretical evolutionary tracks suggests that their photometricallyselected candidates have masses $<0.2 M_{\odot}$ and ages $1-10 \mathrm{Myr}$. Barrado y Navascués \& Jayawardhana (2004) collected spectra for some of these and confirm the presence of a classical $\mathrm{T}$ Tauri object with signatures of disk accretion and outflow near the substellar domain. Cha II has been surveyed during the

\footnotetext{
3 http://iso.esac.esa.int/

4 http://science.nasa.gov/missions/rosat/

5 http://irsa.ipac. caltech.edu/Missions/denis.html
}

course of the Spitzer Legacy program, From Molecular Cores to Planet-forming Disk (c2d; Evans et al. 2003), and as part of the Herschel Gould Belt survey key project (André et al. 2010; Spezzi et al. 2013).

In this work we present very deep observations at $870 \mu \mathrm{m}$ using the LABOCA bolometer at the APEX telescope towards the Cha II complex. The sensitivity of our map $\left(\sim 5 \mathrm{mJy} / 27.6^{\prime \prime}\right.$ beam $)$ provides a $5 \sigma$ threshold for the $\mathrm{H}_{2}$ column density of $2.1 \times$ $10^{21} \mathrm{~cm}^{-2}$, and a $5 \sigma$ mass limit of $\sim 0.015 M_{\odot}$, well within the substellar regime. This is the first time that such a deep map has been undertaken in this region. Our goal is to detect faint cold dust envelopes that surround very low mass objects to provide the best candidates for pre-brown dwarfs (the scaled-down version of the low-mass pre-stellar cores) and proto-brown dwarfs in the Cha II region. We have complemented our submillimetre data with a multiwavelength database, from the optical to the far-infrared, with the aim of revealing the true nature of the detections and to provide clues for their properties and formation mechanism.

This paper is structured as follows: in Sect. 2, we describe the observations and the archival data. In Sect. 3, we show the results. Finally, we present the discussion and the conclusions of this work in Sects. 4 and 5.

\section{Observations and archival data}

\subsection{Selection of the region}

We selected the region in the cloud with most recent star formation to carry out our study. Using InfraRed Array Camera (IRAC) Spitzer archival data, we generated a colour-colour diagram (see Fig. 1, left-hand panel) that was used to classify the stage of the evolution of Cha II members in Class I, II, and III sources, following Allen et al. (2004). Then, we represented the spatial distribution of the known candidate young members of Cha II, and we selected a region of $14^{\prime} \times 14^{\prime}$ projected size where the highest density of very low-mass Class I/II and Class I objects could be observed (see Fig. 1, right-hand 
panel). Two out of the three Class I sources in the area, according to their positions in the colour-colour diagram, lie in the region of our LABOCA observations.

\section{2. $L A B O C A 870 \mu \mathrm{m}$ continuum data}

We carried out continuum observations at $870 \mu \mathrm{m}$ in the selected region using LABOCA bolometer array, installed on the Atacama Pathfinder EXperiment $\left(\mathrm{APEX}^{6}\right)$ telescope. Our data were acquired on 2010 April 08, 09, and 11 during the Onsala Swedish program O-085.F-9301A-2010 under good weather conditions $(p w v \sim 1.20 \mathrm{~mm}$, equivalent to a zenith opacity of 0.34 at $870 \mu \mathrm{m})$. Observations were performed using a spiral raster mapping centred at $\alpha=12^{\mathrm{h}} 53^{\mathrm{m}} 29.9^{\mathrm{s}}, \delta=$ $-77^{\circ} 10^{\prime} 57^{\prime \prime}(\mathrm{J} 2000.0)$ and covering an area of projected size $14^{\prime} \times 14^{\prime}$. The total integration time was $\sim 13 \mathrm{~h}$ ( $10.4 \mathrm{~h}$ on source). The calibration was performed using observations of Mars as well as the secondary calibrator G305.81-0.25. The absolute flux calibration uncertainty was $\sim 8 \%$. The telescope pointing was checked every hour and the focus settings were checked once per night and during the sunset.

Data were reduced using the $\mathrm{CRUSH}^{7}$ software package (see Kovács 2008) and the BOlometer Array Analysis Software $\left(\mathrm{BoA}^{8}\right)$. The preprocessing steps consisted of flagging dead or cross-talking channels, frames with too high telescope accelerations and with unsuitable mapping speed, as well as temperature-drift correction using two blind bolometers. The data reduction process included flat-fielding, opacity correction, calibration, correlated noise removal (atmospherics fluctuations seen by the whole array, as well as electronic noise originated in groups of detector channels), and de-spiking. Every scan was visually inspected to identify and discard corrupted data.

Data processing was optimized to recover faint sources. The final map was smoothed to a final angular resolution of $27.6^{\prime \prime}$. As a result, we obtained a $1 \sigma$ point source sensitivity of $\simeq 4 \mathrm{mJy}$ at the centre of the map and $\simeq 6 \mathrm{mJy}$ at the edges.

\subsection{Mining the archives: optical to far-infrared data}

To properly classify the sources detected at $870 \mu \mathrm{m}$ and search for optical to far-infrared counterparts, we built an extensive multiwavelength catalogue in the Cha II region with public data from different space missions and ground-based telescopes. We used optical data from the Wide Field Imager $\left(\mathrm{WFI}^{9}\right)$ at MPG/ESO $2.2 \mathrm{~m}$ telescope, as well as the Visible Multi-Object Spectrograph $\left(\right.$ VIMOS $\left.^{10}\right)$ in the imaging mode at the VLT.

In the near-infrared, we used the point-source catalogue from the 2 Microns All sky survey (2MASS ${ }^{11}$ ) and the Deep Near Infrared Survey of the Southern Sky (DENIS). The Preliminary Release Image and Source data product from the Wide-field Infrared Survey Explorer (WISE ${ }^{12}$ ) mission,

\footnotetext{
6 This work is partially based on observations with the APEX telescope. APEX is a collaboration between the Max Plank Institute fur Radioastronomie, the European Southern Observatory, and the Onsala Space Observatory.

7 http: //www . submm. caltech. edu/ sharc/crush/

8 http://www.apex-telescope.org/bolometer/laboca/boa/

9 http://www .eso.org/sci/facilities/lasilla/ instruments/wfi.html

${ }^{10}$ http://www . eso.org/sci/facilities/paranal/ instruments/vimos.html

11 http://irsa.ipac. caltech.edu/Missions/2mass.html

12 http://WWw .nasa.gov/mission_pages/WISE/main/index . html
}

Spitzer/IRAC ${ }^{13}$ data and AKARI ${ }^{14}$ Infrared Camera (IRC) Point Source Catalogue data products were considered in the mid-infrared range. Data in the far-infrared were taken from the Multiband Imaging Photometer for Spitzer (MIPS ${ }^{15}$ ) and from the AKARI Far-Infrared Surveyor (FIR) Bright Source Catalogue (see Table 1 for a detailed description). Herschel PACS $^{16}$ and SPIRE ${ }^{17}$ maps were also processed to check the spatial coincidence of the cores that were detected with LABOCA in the filamentary and clumpy structure observed in Herschel maps.

\section{Results}

\subsection{Submillimetre emission at $870 \mu \mathrm{m}$}

The LABOCA map reveals a clumpy distribution of cold dust with two bright sources spatially coincident with well-known YSOs (see Fig. 2). The northern one, known as DK Cha, is associated with IRAS 12496-7650 and the southern one is the submillimetre counterpart of IRAS 12500-7658. Both sources are surrounded by more extended diffused emission, probably tracing material from the parental cloud.

The central part of the map, which is the region with the lowest noise, shows various faint cores. We have identified a total of 15 local maxima at more than $5 \sigma$ emission (see Table 2 for a summary of the main properties of the detections). Eight of them correspond to unresolved point-like sources and the other seven cores show a more extended morphology.

\subsection{Searching for optical to infrared counterparts}

We used our extensive multiwavelength catalogue to search for counterparts to the detections at $870 \mu \mathrm{m}$ (see some examples in Table A.1. The position of every submillimetre source was cross-matched with this catalogue, taking into consideration all possible counterpart candidates that fall into the beam size of LABOCA (27.6") for point-like sources, and into the total core area for the ones showing spatial structure. Once we had identified all the counterpart candidates as a function of the separation to the submillimetre core, we analysed their spectral energy distribution (SED) to identify and classify reliable candidates. An extended explanation of the used method is provided in Appendix A. Results from this method are shown in Table 2.

\subsection{Mass of the cores}

For the calculation of the mass of the cores, we assume that the emission at $870 \mu \mathrm{m}$ comes totally from thermal dust and is optically thin. We estimate the total mass (gas+dust) by assuming a gas-to-dust ratio of 100 and adopting the following relation:

$$
M=\frac{S_{870} d^{2}}{\kappa_{870} B_{870}\left(T_{d}\right)},
$$

\footnotetext{
13 http://www.spitzer.caltech.edu/mission/

398-The-Infrared-Array-Camera-IRAC-

14 http://irsa.ipac.caltech.edu/Missions/akari.html

15 http://www. spitzer.caltech.edu/mission/

396-The-Multiband-Imaging-Photometer-MIPS-

16 http: //www . mpe.mpg.de/ir/Pacs

17 http://www . astro.cardiff.ac.uk/research/astro/instr/ projects $/$ ?page $=$ spire
} 


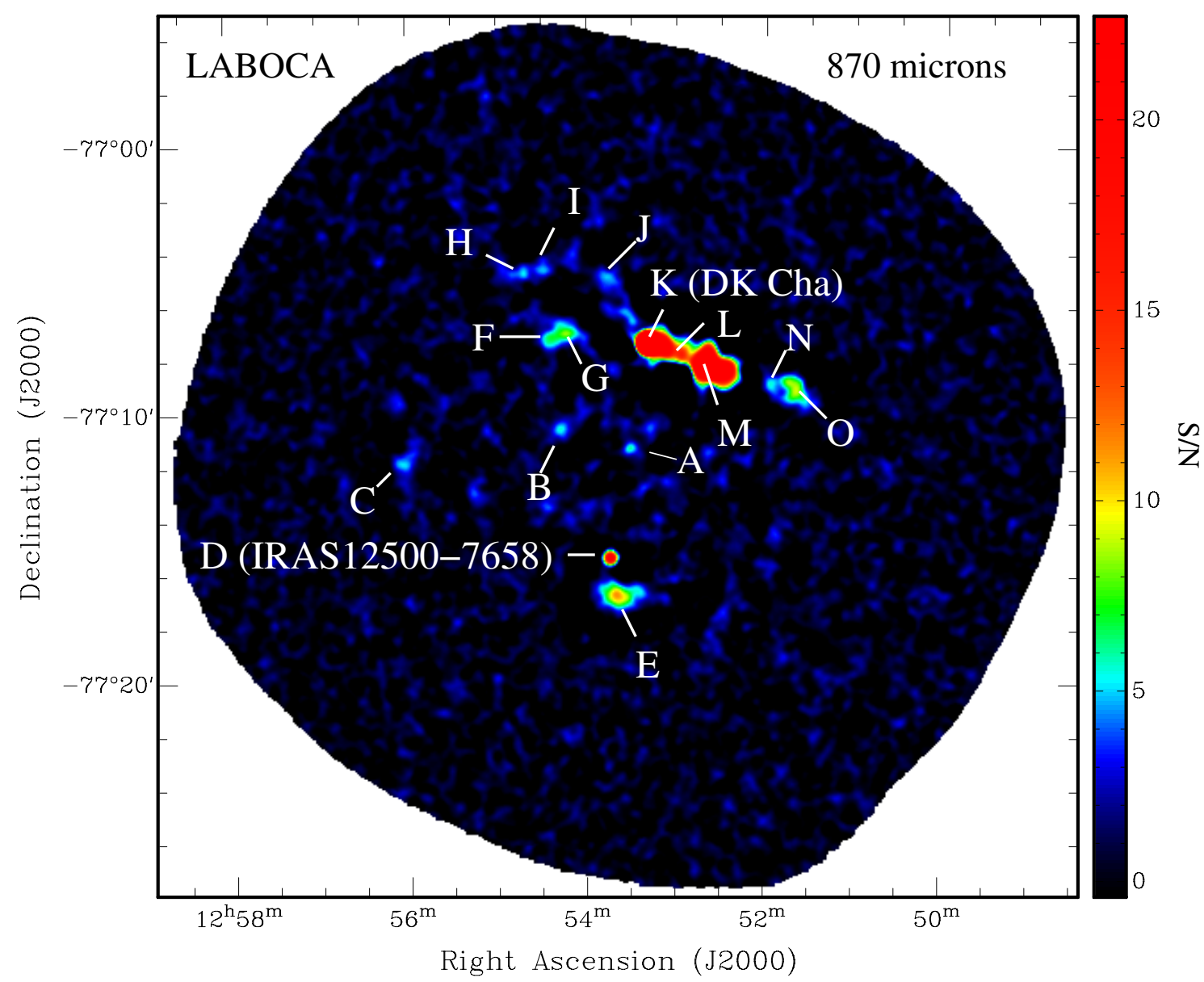

Fig. 2. $\mathrm{S} / \mathrm{N}$ emission map at $870 \mu \mathrm{m}$ observed with LABOCA in the selected area of Cha II region.

Table 1. Archival data used to perform a multiwavelength study in the cores detected at $870 \mu \mathrm{m}$.

\begin{tabular}{|c|c|c|c|c|c|}
\hline $\begin{array}{l}\text { Telescope/ } \\
\text { instrument or survey }\end{array}$ & Filter $_{1}\left(\lambda_{1}\right)$ & Filter $_{2}\left(\lambda_{2}\right)$ & Filter $_{3}\left(\lambda_{3}\right)$ & Filter $_{4}\left(\lambda_{4}\right)$ & Filter $_{5}\left(\lambda_{5}\right)$ \\
\hline ESO $2.2 \mathrm{~m} / \mathrm{WFI}^{a}$ & $R c(651.7 \mathrm{~nm})$ & $\mathrm{H} \alpha(658.8 \mathrm{~nm})$ & NB665 (665.6 nm) & $S I I(676.3 \mathrm{~nm})$ & $I c(783.8 \mathrm{~nm})$ \\
\hline ESO $2.2 \mathrm{~m} / \mathrm{WFI}^{a}$ & $I(826.9 \mathrm{~nm})$ & $M B 856(856.2 \mathrm{~nm})$ & MB914 (914.8 nm) & $Z(964.8 \mathrm{~nm})$ & \\
\hline VLT/VIMOS $^{a}$ & $I(817.1 \mathrm{~nm})$ & & & & \\
\hline ESO $1 \mathrm{~m} / \mathrm{DENIS}$ & $I(0.8 \mu \mathrm{m})$ & $J(1.2 \mu \mathrm{m})$ & $K s(2.1 \mu \mathrm{m})$ & & \\
\hline Mt. Hopkins-CTIO/2MASS & $J(1.2 \mu \mathrm{m})$ & $H(1.7 \mu \mathrm{m})$ & $K(2.2 \mu \mathrm{m}))$ & & \\
\hline WISE & $W 1(3.4 \mu \mathrm{m})$ & $W 2(4.6 \mu \mathrm{m})$ & $W 3(12 \mu \mathrm{m})$ & $W 4(22 \mu \mathrm{m})$ & \\
\hline Spitzer/IRAC ${ }^{b}$ & $I 1(3.6 \mu \mathrm{m})$ & $I 2(4.5 \mu \mathrm{m})$ & $I 3(5.8 \mu \mathrm{m})$ & $I 4(8.0 \mu \mathrm{m})$ & \\
\hline Spitzer/MIPS ${ }^{b}$ & $M 1(24 \mu \mathrm{m})$ & $M 2(70 \mu \mathrm{m})$ & & & \\
\hline Herschel/PACS ${ }^{c}$ & Blue $(70 \mu \mathrm{m})$ & $\operatorname{Red}(160 \mu \mathrm{m})$ & & & \\
\hline Herschel/SPIRE ${ }^{c}$ & Blue $(250 \mu \mathrm{m})$ & Green $(350 \mu \mathrm{m})$ & $\operatorname{Red}(500 \mu \mathrm{m})$ & & \\
\hline AKARI/IRC & $S 9 W(9 \mu \mathrm{m})$ & $L 18 W(18 \mu \mathrm{m})$ & & & \\
\hline AKARI/FIS & $N 60(65 \mu \mathrm{m})$ & $W I D E-S(90 \mu \mathrm{m})$ & WIDE-L $(140 \mu \mathrm{m})$ & $N 160(160 \mu \mathrm{m})$ & \\
\hline
\end{tabular}

Notes. ${ }^{(a)}$ Archival ESO Projects 2064.I-0559, 67.C-0225(A), 077.C-0339(A), 075.C-0294(C), and 68.C-0311(A), for WFI data, and 072.C0046(B) for VIMOS. ${ }^{(b)}$ These data are part of the legacy project "cores to disks" (c2d; see Evans et al. 2003). ${ }^{(c)}$ Data part of the Herschel guaranteed time key programme "Gould Belt survey" (KPGT_andre_1; PI: Philippe André).

where $S_{870}$ is the flux density of the cores (i.e. the integrated emission over the solid angle) at $870 \mu \mathrm{m}, d$ is the distance to the source, $\kappa_{870}$ is the dust opacity per unit mass (gas+dust) column density at $870 \mu \mathrm{m}$, and $B_{870}\left(T_{\mathrm{d}}\right)$ is the Planck function at $870 \mu \mathrm{m}$ for a dust temperature $T_{\mathrm{d}}$. We adopt a distance of $178 \mathrm{pc}$ (Whittet et al. 1997), a $T_{\mathrm{d}}=10 \mathrm{~K}$, and a dust opacity at $870 \mu \mathrm{m}$ of $0.0175 \mathrm{~cm}^{2} \mathrm{gr}^{-1}$ that results from interpolating the values at 700 and $1000 \mu \mathrm{m}$, which are given in Col. 6 of Table 1 of Ossenkopf \& Henning (1994; corresponding to dust grains with thin ice mantles at a density of $10^{6} \mathrm{~cm}^{-3}$ ). The opacity law of Ossenkopf \& Henning (1994) is consistent with the law inferred from Herschel data for infrared dark clouds (e.g. Lim \& Tan 2014). The value adopted by us at $870 \mu \mathrm{m}$ is $\lesssim 50 \%$ compared to the value adopted by the Herschel Gould Belt Survey 
Table 2. Properties of the submillimetre cores detected at $870 \mu \mathrm{m}$ in Cha II.

\begin{tabular}{|c|c|c|c|c|c|c|c|c|c|c|}
\hline Core & $\begin{array}{c}\mathrm{RA}^{a} \\
(\mathrm{~J} 2000)\end{array}$ & $\begin{array}{c}\operatorname{Dec}^{a} \\
(\mathrm{~J} 2000)\end{array}$ & $\begin{array}{c}S_{870_{\mu m}{ }^{b}} \\
(\mathrm{mJy})\end{array}$ & $\begin{array}{l}\mathrm{rms} \\
(\mathrm{mJy})\end{array}$ & $\begin{array}{l}\text { Mass } \\
\left(M_{\odot}\right)\end{array}$ & $\begin{array}{c}\text { Deconvolved }^{c} \\
\text { ang. size }\end{array}$ & $\begin{array}{c}n_{\text {core }} \\
\left(\mathrm{cm}^{-3}\right)\end{array}$ & $\begin{array}{l}n_{\text {crit }^{d}} \\
\left(\mathrm{~cm}^{-3}\right)\end{array}$ & $\begin{array}{l}\text { Sep. } \\
\text { (") }\end{array}$ & Counterpart? \\
\hline ChaII-APEX-A & $12: 53: 30.5$ & $-77: 11: 07$ & 26 & 5 & 0.016 & Point-like & $\ldots$ & $4.3 \times 10^{7}$ & $7^{\prime \prime}, 10^{\prime \prime}$ & No (Background star) \\
\hline ChaII-APEX-B & $12: 54: 17.3$ & $-77: 10: 23$ & 26 & 5 & 0.016 & Point-like & $\ldots$ & $4.3 \times 10^{7}$ & $15^{\prime \prime}$ & No (Background star) \\
\hline ChaII-APEX-D & $12: 53: 42.6$ & $-77: 15: 11$ & 109 & 5 & 0.066 & Point-like & $\ldots$ & $2.5 \times 10^{6}$ & $1^{\prime \prime}$ & Yes (YSO IRAS 12500-7658) \\
\hline ChaII-APEX-E & $12: 53: 39.0$ & $-77: 16: 35$ & $460^{f}$ & 5 & 0.279 & $75^{\prime \prime}$ & $2.8 \times 10^{4}$ & $1.4 \times 10^{5}$ & $\ldots$ & No \\
\hline ChaII-APEX-F & $12: 54: 21.9$ & $-77: 06: 59$ & 35 & 5 & 0.021 & Point-like & $\ldots$ & $2.5 \times 10^{7}$ & $\ldots$ & No \\
\hline ChaII-APEX-G & $12: 54: 12.3$ & $-77: 06: 47$ & $130^{f}$ & 5 & 0.079 & $45^{\prime \prime}$ & $3.7 \times 10^{4}$ & $1.7 \times 10^{6}$ & $11^{\prime \prime}$ & No (Background star) \\
\hline ChaII-APEX-I & $12: 54: 27.7$ & $-77: 04: 27$ & 30 & 6 & 0.018 & Point-like & $\ldots$ & $3.4 \times 10^{7}$ & $13^{\prime \prime}$ & No (Background star) \\
\hline ChaII-APEX-J & $12: 53: 44.8$ & $-77: 04: 43$ & $39^{f}$ & 6 & 0.024 & $40^{\prime \prime}$ & $1.5 \times 10^{4}$ & $1.9 \times 10^{7}$ & $18^{\prime \prime}$ & No (Background star) \\
\hline ChaII-APEX-K & $12: 53: 17.3$ & $-77: 07: 11$ & 1745 & 4 & 1.059 & Point-like & $\ldots$ & $9.7 \times 10^{3}$ & $0.5^{\prime \prime}$ & Yes (YSO DK Cha) \\
\hline ChaII-APEX-L & $12: 52: 55.8$ & $-77: 07: 35$ & 78 & 5 & 0.047 & Point-like & $\ldots$ & $4.9 \times 10^{6}$ & $1^{\prime \prime}$ & Yes (Proto-BD candidate) \\
\hline ChaII-APEX-M & $12: 52: 39.0$ & $-77: 07: 59$ & $3700^{f}$ & 4 & 2.246 & $80^{\prime \prime}$ & $1.8 \times 10^{5}$ & $2.2 \times 10^{3}$ & $\ldots$ & No \\
\hline ChaII-APEX-N & $12: 51: 55.8$ & $-77: 08: 43$ & 30 & 5 & 0.018 & Point-like & & $3.4 \times 10^{7}$ & $16^{\prime \prime}$ & No (Background star) \\
\hline ChaII-APEX-O & $12: 51: 39.0$ & $-77: 08: 55$ & $260^{f}$ & 6 & 0.158 & $55^{\prime \prime}$ & $4.0 \times 10^{4}$ & $4.4 \times 10^{5}$ & $13^{\prime \prime}, 16^{\prime \prime}$ & No (Background star) \\
\hline
\end{tabular}

Notes. ${ }^{(a)}$ Position of the maximum emission of the cores. Units of right ascension are hours, minutes, and seconds. Units of declination are degrees, arcminutes, and arcseconds. ${ }^{(b)}$ Flux density computed above $3 \sigma$ emission. ${ }^{(c)}$ Deconvolved size after fitting a Gaussian to the cores. The averaged of the major and minor axis is represented. ${ }^{(d)}$ Critical density of the core, calculated following the equation in Sect. 4.4. ${ }^{(e)}$ Separation of the counterpart candidates to the nominal position of the submillimetre core. ${ }^{(f)}$ For cores showing extended emission we applied a specific data processing (using option "extended" in CrUSH) to avoid filtering out extended structures.

(of $\sim 0.01189 \mathrm{~cm}^{2} \mathrm{~g}^{-1}$, Roy et al. 2013), well below the typical uncertainty for the dust opacity inferred from the modelling of Herschel data, by about a factor of two (Wagle et al. 2015).

Flux density was calculated by integrating the emission above $3 \sigma$ contours. The derived core masses are affected by errors that mainly come from the uncertainty in $T_{\mathrm{d}}$, dust opacity, and the distance. The error in the distance calculation is estimated to be $18 \mathrm{pc}$ (Whittet et al. 1997), providing a change in the core masses estimation by a factor $\sim 1.2$. An increment of a factor two in the value of the opacity and of the $T_{\mathrm{d}}$ produces a decrement in the value of the mass of a factor of $\sim 2$ and $\sim 3.3$, respectively. Therefore the highest source of error for the estimation of masses is the value of the dust temperature. Previous works that aimed to model the dust temperature profiles of prestellar cores provided a variation from $13 \mathrm{~K}$ in the outer parts and $7 \mathrm{~K}$ in the inner parts (see Evans et al. 2001). In this work we adopt an average value of $T_{\mathrm{d}}=10 \mathrm{~K}$. The adopted criteria provides 11 cores with masses in the substellar regime and four cores with stellar masses (see Table 2).

\section{Discussion}

\subsection{Nature of the counterpart candidates}

To reveal the nature of the optical to infrared possible counterparts that were found, we performed a study of the SED properties of the submillimetre cores using Robitaille et al. (2006, 2007) SED tool on every reliable counterpart candidate. From this analysis we derive that two cores (D and K) are clearly associated with the well known young objects DK Cha (IRAS 124967650) and IRAS 12500-7658. Nine cores (A, B, C, G, H, I, J, N, and O) lack any YSO candidate counterpart, being the SEDs of the found possible counterparts that were compatible with extincted background stars, and hence not associated with the submillimetre cores. Cores E, F, and M do not show any counterpart. In core L, we found a YSO candidate with counterparts in I1 and I2 IRAC bands. These results are summarised in Table 2.

The NASA/IPAC Extragalactic Database $\left(\mathrm{NED}^{18}\right)$ was checked to search for extragalactic contamination at the position of each core and no extragalactic counterparts were found. We

\footnotetext{
18 https://ned.ipac.caltech.edu/
}

also compared our map with recent public Herschel observations with SPIRE and PACS, obtaining a very good spatial coincidence between the cores at $870 \mu \mathrm{m}$ and the clumpy filament emission shown by Herschel (see Fig. 3 for a close up superposition of every core detected at $870 \mu \mathrm{m}$ with PACS at $160 \mu \mathrm{m}$ and Fig. 4 for an overlap of the SPIRE map at $350 \mu \mathrm{m}$ and the region observed with $\mathrm{LABOCA}$ ). We note the faint dusty microfilaments, where most of the LABOCA cores seem to be embedded. These filaments, observed at a noise level in the LABOCA map and clearly confirmed by Herschel, suggest a scaled-down version of the network of longer and dense filaments recently studied by André et al. (2010), Könyves et al. (2010) and Hacar et al. (2013) in several molecular clouds, which are proposed as being the precursors of prestellar cores via fragmentation.

\subsection{Previously known YSOs in the region}

IRAS 12496-7650, also known as DK Cha, is the most massive object in Cha II region, an intermediate-mass Herbig Ae star in a transitional stage of evolution between Class I and II (Spezzi et al. 2008) that hosts a face-on disk-outflow system (van Kempen et al. 2010). This source has been extensively studied by Spezzi et al. (2013), and provides an estimation of the most probable parameters of the disk surrounding DK Cha. Spezzi et al. (2013) model the SED of this source from the optical to millimetre wavelengths (using Herschel data), while the flux at $870 \mu \mathrm{m}$, which is expected from their set of bestfitting models, agrees very well with our measurements given in Table 2.

Our deep submillimetre observations reveal this source immersed in a well-defined elongated and clumpy nebulosity. Elongated structures with multiple sub-peaks have been observed surrounding other intermediate-mass YSOs, because it is not clear whether the source of this emission is due to multiple sources embedded in the elongated structure, to shocks in the outflow, or to just tracing the natal cloud (e.g. Fuente et al. 2007; Rathborne et al. 2005). In the case of DK Cha, it has been proposed that the outflow is perpendicular to the plane of the sky, which rules out this phenomenon as the cause of the observed dust structure. Taking into consideration the filamentary structures that were observed in Herschel maps, and 

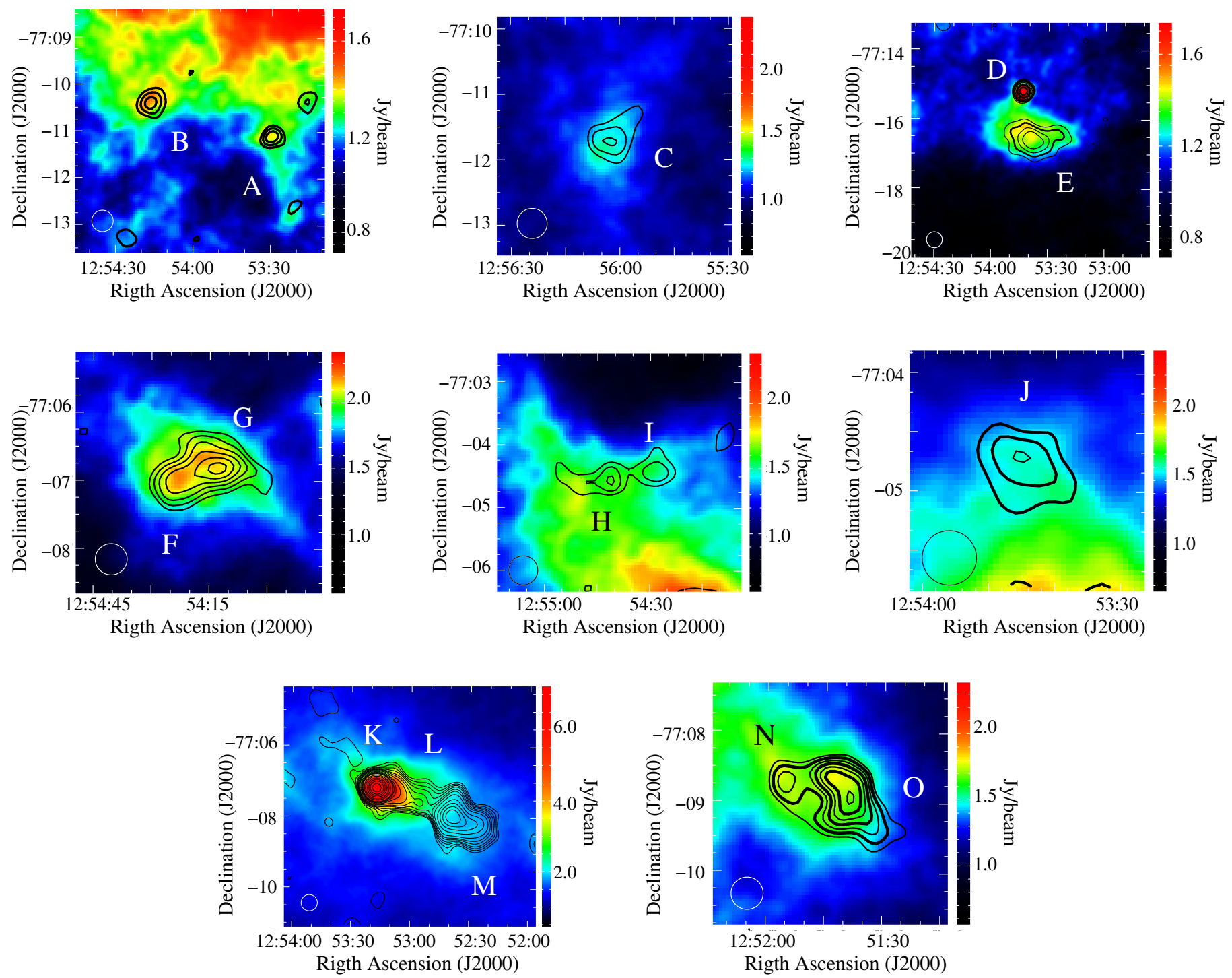

Fig. 3. Close-up with details of the submillimetre cores with a $S / N \geq 5$. Colours represent the Herschel PACS map at $160 \mu$ m superposed onto the background. Contours represent LABOCA emission at $870 \mu \mathrm{m}$. Lower contour represents $3 \sigma$ emission, with an increment of $1 \sigma$ between contours for all cores, except for the one corresponding to cores K (DK Cha), L, and M, with contours at $3 \sigma, 5 \sigma, 7 \sigma, 9 \sigma, 12 \sigma, 15 \sigma, 20 \sigma, 30 \sigma, 40 \sigma, 50 \sigma$, $60 \sigma, 90 \sigma, 120 \sigma, 170 \sigma, 220 \sigma, 270 \sigma, 350 \sigma$, and cores D (IRAS 12500-7658) and E, with contours at $3 \sigma, 5 \sigma, 7 \sigma, 9 \sigma, 12 \sigma, 15 \sigma$, and $20 \sigma$. See different range of values of root mean square (rms) in Table 2.

that have multiple cores embedded inside, this type of stream of dust clumps may be tracing the embedded YSOs that are being formed inside the natal cloud.

Besides DK Cha there is another young central object in the elongated dust structure, which corresponds to the infrared counterpart of core L (see Sect. 4.3). A high level of fragmentation is revealed in our map and one of the embedded cores (core M) seems to be gravitationally unstable (see Sect. 4.4). We therefore conclude that they are multiple pre-stellar and protostellar cores belonging to the same parental nebula.

IRAS 12500-7658 has been catalogued as a Class I object of spectral type close to K5 (Spezzi et al. 2013). In this work, we detect compact submillimetre emission that is associated with this IRAS source and that is very close to a southern and extended starless core of $\simeq 10^{4} \mathrm{AU}$ size (see Figs. 2 and 4 ), the scale of dense cores being typical of regions where low-mass stars are formed. Spezzi et al. (2013) modelled in detailed its SED and inferred a first estimation of the parameters of the young disk that surrounds this source, predicting a flux at 870 micron, which is consistent with our observed value (Table 2). No further analysis will be presented in this work beyond the SED and the photometry shown in Fig. A.1 and Table A.1.

\subsection{A possible candidate for proto-BD}

The infrared counterpart (I1, I2) of core L lies only about $\sim 1^{\prime \prime}$ away from the peak of the submillimetre source. This excellent match, which can be seen in Fig. 5 (left), strongly suggests that the infrared and submillimetre sources are spatially associated. To further explore the possible nature of core L, we compared its SED (we list its photometry in Table 3) to the known SEDs of previously reported Class I (J041757B, Barrado et al. 2009; Palau et al. 2012) and Class 0 (Lee et al. 2013; Palau et al. 2014) proto-BD candidates, and the result is shown in Fig. 5 (right). The figure shows that the IRAC and $870 \mu \mathrm{m}$ fluxes are comparable to the fluxes of the Class I proto-BD candidate J041757B. But core L could still be a Class 0 proto-BD, depending on the real values of the fluxes between 5 and $500 \mu \mathrm{m}$, 


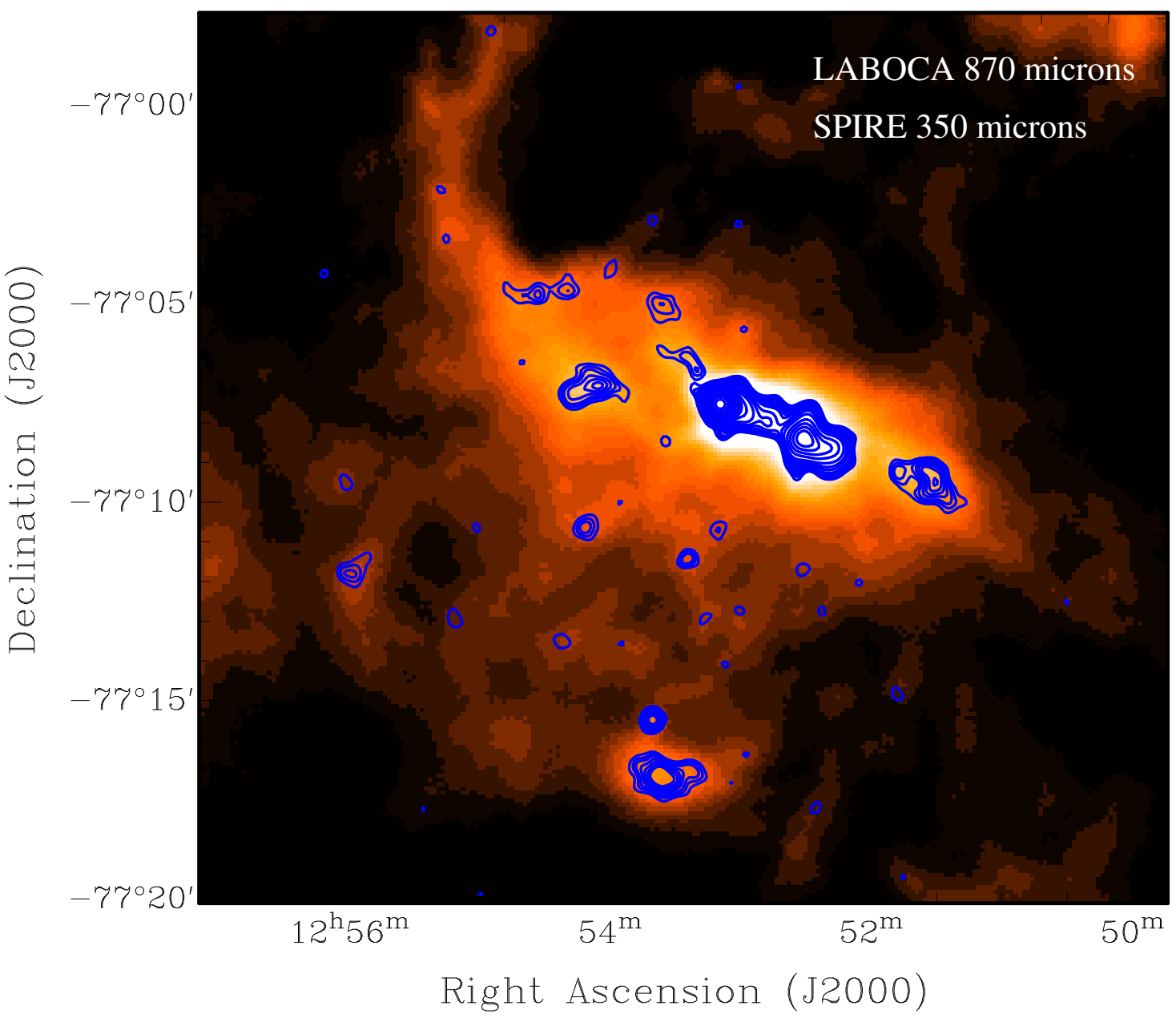

Fig. 4. Herschel/SPIRE emission map at $350 \mu \mathrm{m}$ (colour) superposed with LABOCA emission at $870 \mu \mathrm{m}$ (contours; levels similar to DK Cha panel in Fig. 2).

for which only the upper limits could be estimated because of the proximity of DK Cha, which dominates the emission at these wavelengths. The upper limit to the bolometric luminosity of core $\mathrm{L}$ is $<0.08 L_{\odot}$, which is similar to the bolometric luminosity of the Class 0 proto-BD IC348-SMM2E (Palau et al. 2014). Such a low bolometric luminosity, together with the fact that its envelope mass is substellar $\left(\sim 50 M_{\text {Jup }}\right)$, indicates that core $\mathrm{L}$ could be a proto-BD candidate. However, this is still a speculative result based on three photometry points (in the near infrared and at submillimetre frequencies) and a set of upper limits, and because of the scarce number of known proto-BD candidates, the core ChaII-APEX-L deserves further investigation to confirm its real nature.

\subsection{Cores with no counterpart: pre-BD cores?}

Padoan \& Nordlund (2004), who investigated the in situ formation of BDs in supersonically turbulent clouds via turbulent fragmentation, stated that substellar objects can be formed in cores with a density as high as the critical one for the collapse of a BD mass core. These authors used the mass of the critical Bonnor-Ebert isothermal sphere to infer the critical density:

$n_{\text {crit }}=\left(\frac{3.3}{M_{\mathrm{BE}}}\right)^{2}\left(\frac{T}{10}\right)^{3} 10^{3}$,

with $M_{\mathrm{BE}}$ in $M_{\odot}, T$ in $\mathrm{K}$, and $n_{\text {crit }}$ in $\mathrm{cm}^{-3}$.
Table 3. Photometry for core L.

\begin{tabular}{cccl}
\hline \hline $\begin{array}{c}\lambda \\
(\mu \mathrm{m})\end{array}$ & $\begin{array}{c}S_{v} \\
(\mathrm{mJy})\end{array}$ & $\begin{array}{c}\sigma \\
(\mathrm{mJy})\end{array}$ & Instrument \\
\hline 3.6 & 0.276 & 0.025 & Spitzer/IRAC \\
4.5 & 0.29 & 0.04 & Spitzer/IRAC \\
5.8 & $<1.3$ & $\ldots$ & Spitzer/IRAC \\
8.0 & $<1.0$ & $\ldots$ & Spitzer/IRAC \\
70 & $<108$ & $\ldots$ & Herschel/PACS \\
160 & $<2290$ & $\ldots$ & Herschel/PACS \\
250 & $<2050$ & $\ldots$ & Herschel/SPIRE \\
350 & $<2310$ & $\ldots$ & Herschel/SPIRE \\
500 & $<2610$ & $\ldots$ & Herschel/SPIRE \\
870 & 78 & 4.9 & APEX/LABOCA \\
\hline
\end{tabular}

For the resolved extended starless cores C, E, G, H, J, and O, we obtain a $n_{\text {core }}<n_{\text {crit }}$ (see Table 2 ), which suggests that all of them are gravitationally stable transient cores. Core M, close to DK Cha and with a mass in the stellar regime, is the only one that presents extended emission with a $n_{\text {core }}>n_{\text {crit }}$, with a value of $n_{\text {core }}=1.8 \times 10^{5} \mathrm{~cm}^{-3}$ two orders of magnitude larger than $n_{\text {crit }}=2.2 \times 10^{3} \mathrm{~cm}^{-3}$. This result suggests that this core is in a pre-stellar, gravitationally unstable, stage.

For the rest of the point-like (unresolved) sources detected in our APEX/LABOCA map, starless (cores A, B, F, I, and N) or 

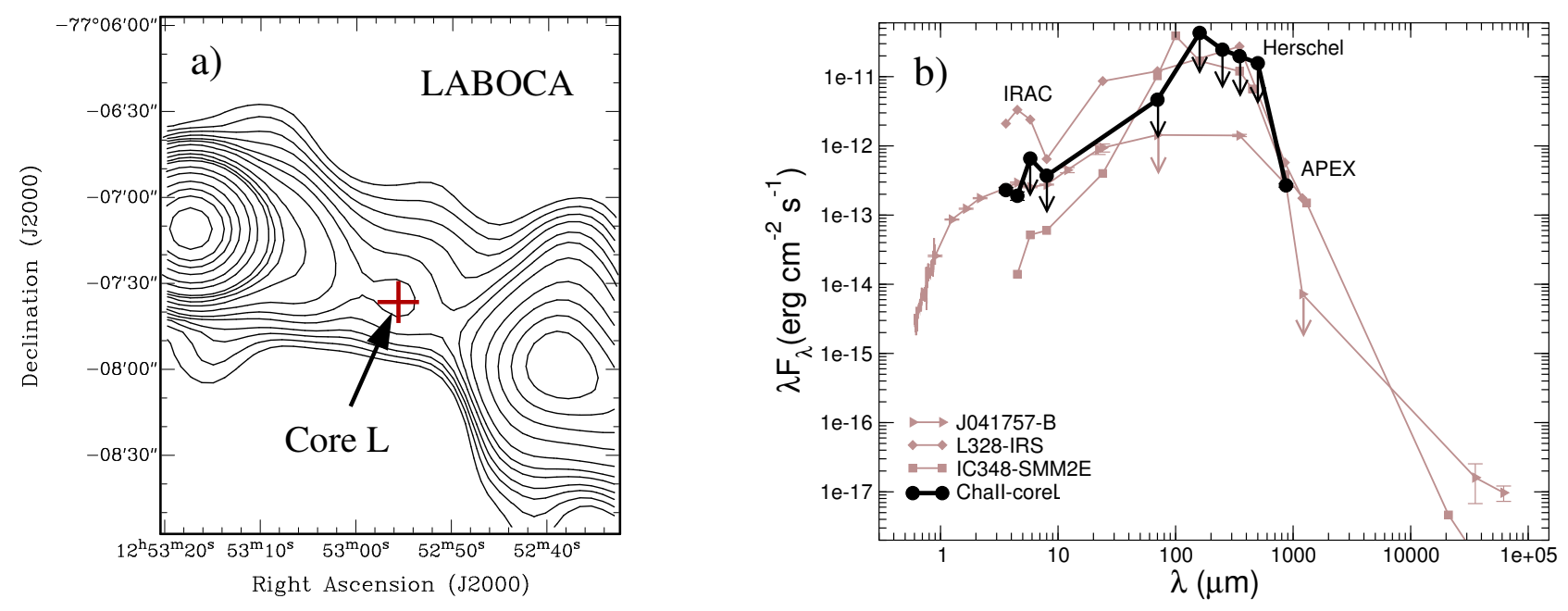

Fig. 5. a) Close-up of LABOCA emission at core L (similar contour levels as in Fig. 2). Red cross represents the position of the IRAC 2 Spitzer emission at the position of core L. b) Spectral energy distribution of core L (black thick line) and the proto-BD candidates J041757-B, L328-IRS, and IC348-SMM2E (brown thin lines; Palau et al. 2012, 2014; Lee et al. 2013). Arrows represent upper limits.

associated with a central YSO (cores D,K, and L), we calculated the maximum radius required to have a core density higher than the critical one. We conclude that for objects in the sub-stellar regime, a radius of between $R \leq 220$ AU for the less massive cores $\left(0.016 M_{\odot}\right)$ and $R \leq 907$ AU for the most massive one $\left(0.066 M_{\odot}\right)$ are needed to become gravitationally unstable.

Whether these faint starless point-like sources are transient cores or will grow in mass and become pre-stellar objects that form brown dwarfs or/and planetary-mass objects can only be answered by studies at high-angular resolution and highsensitivity using a telescope like ALMA (a radius of $220 \mathrm{AU}$ at $178 \mathrm{pc}$ subtends a projected size of $1.2^{\prime \prime}$, and ALMA can achieve a spatial resolution below $0.1^{\prime \prime}$ at the frequencies of the study presented here). In addition, spectroscopic observations similar to those reported by André et al. (2012), who use the Plateau de Bure interferometer to observe the now confirmed pre-brown dwarf Oph B-11, will provide the final answer to their energetic state and their dynamical mass.

\section{Conclusions}

In this work we present high-sensitivity observations at $870 \mu \mathrm{m}$ toward an active star-forming region in the Chamaeleon II molecular cloud, which provides a $5 \sigma$ mass limit of $0.015 M_{\odot}$ in the substellar regime. Our main goal was to identify a population of pre- and proto-brown dwarf candidates, which could be studied in detail with future ALMA observations. We built an extensive multiwavelength catalogue from the optical to the far-infrared to search for counterparts and classify the sources detected at $870 \mu \mathrm{m}$ properly. The main conclusions are:

- A total of 15 cores were detected in the mapped region at $870 \mu \mathrm{m}, 11$ of which have substellar masses and four of which show masses in the stellar regime.

- Two cores are the submillimetre counterparts of the well known YSOs DK Cha and IRAS 12500-7658. We identified a possible candidate to proto-BD (core ChaII-APEX-L) that shows counterparts at 3.6 and $4.5 \mu \mathrm{m}$. The rest of the cores have a starless nature, with seven of them showing extended emission and five being spatially unresolved, pointlike sources.
- Based on the Padoan \& Nordlund (2004) theory of formation of BDs via turbulent fragmentation, we infer that most of the spatially resolved cores are transient and gravitationally stable, except core ChaII-APEX-M, the density of which is higher than the critical one. For point-like starless cores in the substellar regime (with masses between $0.016 M_{\odot}$ and $0.066 M_{\odot}$ ) we conclude that radii smaller than $220 \mathrm{AU}$ to 907 AU, respectively, are needed to be in a gravitationally unstable stage to became pre-brown dwarfs.

This paper, based on very deep observations at $870 \mu \mathrm{m}$, provides results that are based on high sensitivity data that complements the Herschel maps and illustrates the role of ground-based submillimetre telescopes in the identification of candidates for future ALMA studies on the formation of young substellar mass objects. We also highlight the need for multiwavelength studies to interpret the results properly.

Acknowledgements. I.d.G. acknowledge support from MICINN (Spain) AYA 2011-30228-C03 grant (including FEDER funds). H.B. is funded by the Ramón y Cajal fellowship program number RYC-2009-04497. A.B. acknowledges financial support from the Proyecto Fondecyt Iniciación 11140572. A.P. acknowledges the financial support from UNAM, and CONACyT (México). C.E. is partly supported by Spanish Grants AYA 2011-26202 and AYA 201455840-P. I.d.G. acknowledges APEX staff for their hard work and support during the LABOCA observation. This publication makes use of data products from the Wide-field Infrared Survey Explorer, Two Micron All Sky Survey, the Spitzer Space Telescope, Herschel and AKARI. This research has been funded by Spanish grants AYA 2012-38897-C02-01 and PRICIT-S2009/ESP-1496.

\section{References}

Alcalá, J. M., Covino, E., Sterzik, M. F., et al. 2000, A\&A, 355, 629 Alcalá, J. M., Spezzi, L., Chapman, N., et al. 2008, ApJ, 676, 427 André, P., Ward-Thompson, D., \& Barsony, M. 1993, ApJ, 406, 122 André, P., Ward-Thompson, D., \& Barsony, M. 2000, Protostars and Planets IV, 59

André, P., Men'shchikov, A., Bontemps, S., et al. 2010, A\&A, 518, L102

André, P., Ward-Thompson, D., \& Greaves, J. 2012, Science, 337, 69

Allen, L. E., Calvet, N., D'Alessio, P., et al. 2004, ApJS, 154, 363

Barrado, D., Morales-Calderón, M., Palau, A., et al. 2009, A\&A, 508, 859

Barrado y Navascués, D., \& Jayawardhana, R. 2004, ApJ, 615, 840 Bate, M. R. 2012, MNRAS, 419, 3115

Bate, M. R., Bonnell, I. A., \& Bromm, V. 2002, MNRAS, 332, L65

Bonnor, W. B. 1956, MNRAS, 116, 351

Epchtein, N., de Batz, B., Capoani, L., et al. 1997, The Messenger, 87, 27 
I. de Gregorio-Monsalvo et al.: A search for pre- and proto-brown dwarfs in Chamaeleon II

Evans, N. J., II, Rawlings, J. M. C., Shirley, Y. L., \& Mundy, L. G. 2001, ApJ, 557,193

Evans, N. J., II, Allen, L. E., Blake, G. A., et al. 2003, PASP, 115, 965

Fuente, A., Ceccarelli, C., Neri, R., et al. 2007, A\&A, 468, L37

Greaves, J. S., Holland, W. S., \& Pound, M. W. 2003, MNRAS, 346, 441

Gueth, F., Bachiller, R., \& Tafalla, M. 2003, A\&A, 401, L5

Hacar, A., Tafalla, M., Kauffmann, J., \& Kovács, A. 2013, A\&A, 554, A55

Hartigan, P. 1993, AJ, 105, 1511

Könyves, V., André, P., Men'shchikov, A., et al. 2010, A\&A, 518, L106

Kovács, A. 2008, Proc. SPIE, 7020

Lada, C. J. 1987, Star Forming Regions, 115, 1

Larson, K. A., Whittet, D. C. B., Prusti, T., \& Chiar, J. E. 1998, A\&A, 337, 465

Lee, C. W., Kim, M.-R., Kim, G., et al. 2013, ApJ, 777, 50

Lim, W., \& Tan, J. C. 2014, ApJ, 780, L29

Maury, A. J., André, P., Hennebelle, P., et al. 2010, A\&A, 512, A40

Ossenkopf, V., \& Henning, T. 1994, A\&A, 291, 943

Padoan, P., \& Nordlund, A. 2004, ApJ, 617, 559

Palau A., de Gregorio-Monsalvo, I., Morata, O., et al. 2012, MNRAS, 424, 2778
Palau, A., Zapata, L. A., Rodríguez, L. F., et al. 2014, MNRAS, submitted Persi, P., Marenzi, A. R., Gómez, M., \& Olofsson, G. 2003, A\&A, 399, 995 Rathborne, J. M., Jackson, J. M., Chambers, E. T., et al. 2005, ApJ, 630, L181 Reipurth, B., \& Clarke, C. 2001, AJ, 122, 432

Robitaille, T. P., Whitney, B. A., Indebetouw, R., Wood, K., \& Denzmore, P. 2006, ApJS, 167, 256

Robitaille, T. P., Whitney, B. A., Indebetouw, R., \& Wood, K. 2007, ApJS, 169, 328

Roy, A., Martin, P. G., Polychroni, D., et al. 2013, ApJ, 763, 55

Schwartz, R. D. 1977, ApJS, 35, 161

Spezzi, L., Alcalá, J. M., Covino, E., et al. 2008, ApJ, 680, 1295

Spezzi, L., Cox, N. L. J., Prusti, T., et al. 2013, A\&A, 555, A71

Stamatellos, D., \& Whitworth, A. P. 2009, MNRAS, 392, 413

van Kempen, T. A., Green, J. D., Evans, N. J., et al. 2010, A\&A, 518, L128

Vuong, M. H., Cambrésy, L., \& Epchtein, N. 2001, A\&A, 379, 208

Wagle, G. A., Troland, T. H., Ferland, G. J., \& Abel, N. P. 2015, ApJ, 809, 17

Whittet, D. C. B., Prusti, T., Franco, G. A. P., et al. 1997, A\&A, 327, 1194

Whitworth, A. P., \& Zinnecker, H. 2004, A\&A, 427, 299 


\section{Appendix A: Identification of counterparts}

To identify counterparts of the submillimetre cores that were detected with LABOCA at other wavelengths, we performed an $\mathrm{X}$-matching procedure, using the following strategy: We took as the base for the $\mathrm{X}$-matching radius the one that was derived from the LABOCA beam and we proceeded in pairs by crossing this LABOCA parental catalog with each catalog that corresponds to a different frequency and/or instrument (individual catalogs corresponding to: 2MASS, AKARI, DENIS, Spitzer/IRAC, channels 1-4 independently, Spitzer/MIPS, Channels 1 and 2 independently, WFI and VLT/VIMOS; and finally WISE; see Table 1). Herschel/SPIRE and PACS maps were not used to identify counterparts because of the clumpy nature of their emission. Nevertheless these data were crucial when confirming the detection of the faintest cores observed in our LABOCA map.
Once we had each possible counterpart for each match, we visually inspected their positions in the IRAC, WISE, and 2MASS images and discarded all possible counterparts that corresponded to artifacts in the images (areas that compromised saturation, bright star spikes, ghosts, etc.). For the counterparts that passed the first visual inspection of images, we performed a combined analysis of the counterparts' astrometry, grouping those objects whose positions at all different wavelengths are compatible with each other, given the typical uncertainty of the positions in those catalogs, which is much smaller than the size of the LABOCA beam, see Fig. A.1. To reveal the nature of the counterparts that were found, we studied the SED properties using Robitaille's SED tool (Robitaille et al. 2006, 2007), which allowed us to differentiate between background stars and YSOs in the found counterpart candidates.
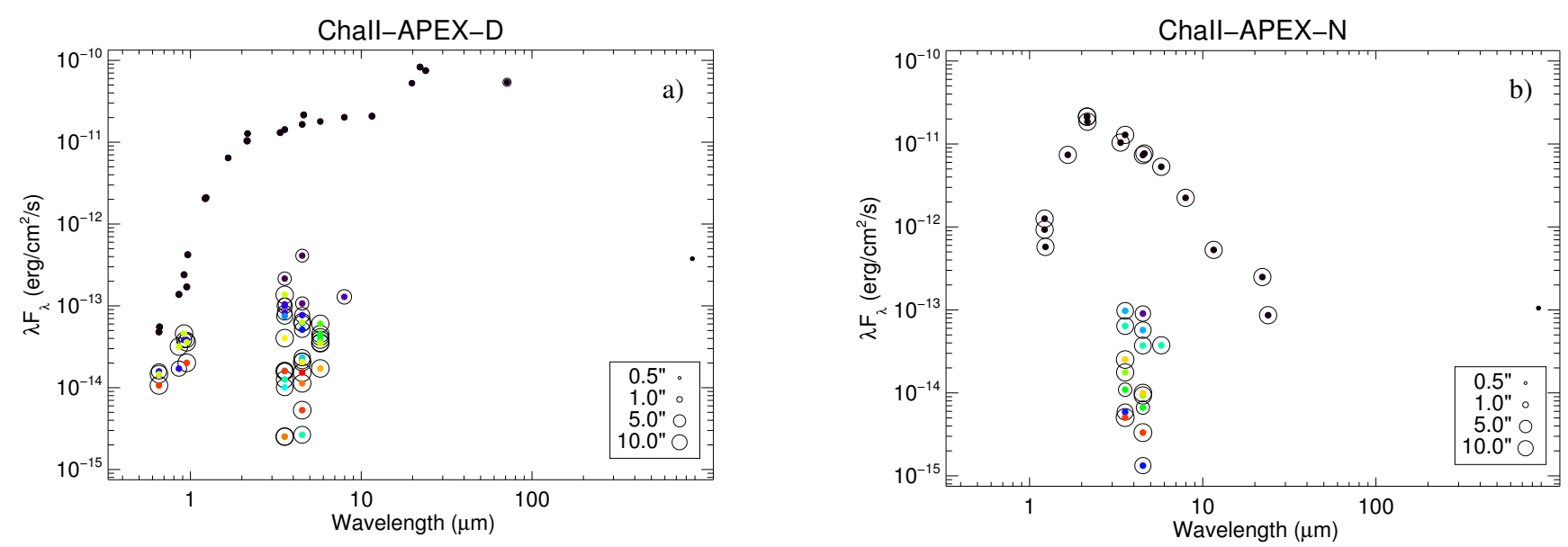

Fig. A.1. Flux versus wavelength of all the counterpart candidates found for core D a) and core N b). Dots of different colours represent different counterpart candidates that have the same spatial position (after taking pointing errors into consideration). The distance of the counterpart candidates to the nominal position of the submillimetre cores is given by the size of the black circle that surrounds each coloured dot. At the right bottom of each figure a) and b), a diagram with the equivalence between the black circle radius and the distance to the cores is indicated. For Core D, the closest counterpart candidate has an SED typical of a YSO, while the rest of the sources located at other more distant positions show no clear nature. In Core N, no YSO is associated and only a source with an SED typical of an extinct background star (black dots; and hence not associated with the submillimetre core) is found. 
I. de Gregorio-Monsalvo et al.: A search for pre- and proto-brown dwarfs in Chamaeleon II

Table A.1. Photometry for the YSO counterpart found for Clumps D and K, and for the background star that is spatially coincident with Clump N.

\begin{tabular}{|c|c|c|c|c|c|c|c|c|}
\hline $\begin{array}{l}\text { Telescope/ } \\
\text { Instrument or survey }\end{array}$ & Filter & $\begin{array}{c}\lambda \\
(\mu \mathrm{m})\end{array}$ & $\begin{array}{l}S_{v}(\mathrm{D}) \\
(\mathrm{Jy})\end{array}$ & $\begin{array}{c}\sigma(\mathrm{D}) \\
(\mathrm{Jy})\end{array}$ & $\begin{array}{l}S_{v}(\mathrm{~N}) \\
(\mathrm{Jy})\end{array}$ & $\begin{array}{c}\sigma(\mathrm{N}) \\
(\mathrm{Jy})\end{array}$ & $\begin{array}{c}S_{v}(\mathrm{DK} \text { Cha }) \\
(\mathrm{Jy})\end{array}$ & $\begin{array}{c}\sigma(\mathrm{DK} \text { Cha }) \\
(\mathrm{Jy})\end{array}$ \\
\hline ESO $2.2 \mathrm{~m} / \mathrm{WFI}$ & $R_{\mathrm{c}}$ & 0.65 & $1.05 \mathrm{e}-05$ & $5.61 \mathrm{e}-07$ & $\ldots$ & $\ldots$ & $7.37 \mathrm{e}-04$ & $1.18 \mathrm{e}-06$ \\
\hline ESO $2.2 \mathrm{~m} / \mathrm{WFI}$ & $\mathrm{H}_{\alpha}$ & 0.66 & $1.22 \mathrm{e}-05$ & $1.39 \mathrm{e}-06$ & $\ldots$ & $\ldots$ & $1.05 \mathrm{e}-03$ & $3.89 \mathrm{e}-06$ \\
\hline ESO $2.2 \mathrm{~m} / \mathrm{WFI}$ & NB665 & 0.67 & $\ldots$ & $\ldots$ & $\ldots$ & $\ldots$ & $6.72 \mathrm{e}-04$ & $2.97 \mathrm{e}-06$ \\
\hline ESO $2.2 \mathrm{~m} / \mathrm{WFI}$ & MB856 & 0.86 & $3.94 \mathrm{e}-05$ & $2.01 \mathrm{e}-06$ & $\ldots$ & $\ldots$ & $9.63 e-03$ & $1.21 \mathrm{e}-05$ \\
\hline ESO $2.2 \mathrm{~m} / \mathrm{WFI}$ & MB914 & 0.92 & $7.34 \mathrm{e}-05$ & $1.71 \mathrm{e}-06$ & $\ldots$ & $\ldots$ & $1.03 e-02$ & $1.35 \mathrm{e}-05$ \\
\hline ESO $2.2 \mathrm{~m} / \mathrm{WFI}$ & Z & 0.96 & $1.36 \mathrm{e}-04$ & $6.63 e-06$ & $\ldots$ & $\ldots$ & $1.72 \mathrm{e}-02$ & $2.54 \mathrm{e}-05$ \\
\hline ESO $2.2 \mathrm{~m} / \mathrm{WFI}$ & $I_{\mathrm{c}}$ & 0.96 & $5.41 \mathrm{e}-05$ & $1.03 \mathrm{e}-06$ & $\ldots$ & $\ldots$ & $6.78 \mathrm{e}-03$ & $7.64 \mathrm{e}-06$ \\
\hline ESO $1 \mathrm{~m} / \mathrm{DENIS}$ & $J$ & 1.2 & $8.37 \mathrm{e}-04$ & $1.47 \mathrm{e}-04$ & $5.13 e-04$ & $1.18 \mathrm{e}-04$ & $1.27 \mathrm{e}-01$ & $5.85 \mathrm{e}-03$ \\
\hline ESO $1 \mathrm{~m} / \mathrm{DENIS}$ & $K_{\mathrm{s}}$ & 2.1 & $7.45 \mathrm{e}-03$ & $8.23 \mathrm{e}-04$ & $1.52 \mathrm{e}-02$ & $1.26 \mathrm{e}-03$ & 5.84 & $4.83 e-01$ \\
\hline ESO $1 \mathrm{~m} / \mathrm{DENIS}$ & $I$ & 0.8 & 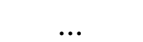 & $\ldots$ & $\ldots$ & $\ldots$ & $3.15 \mathrm{e}-03$ & $1.16 \mathrm{e}-04$ \\
\hline Mt. Hopkins-CTIO/2MASS & $J$ & 1.2 & $8.69 \mathrm{e}-04$ & $6.32 \mathrm{e}-05$ & $2.37 \mathrm{e}-04$ & $4.58 \mathrm{e}-05$ & $2.98 \mathrm{e}-01$ & $6.04 \mathrm{e}-03$ \\
\hline Mt. Hopkins-CTIO/2MASS & $H$ & 1.7 & $3.56 \mathrm{e}-03$ & $1.38 \mathrm{e}-04$ & $4.10 \mathrm{e}-03$ & $1.13 \mathrm{e}-04$ & 1.62 & $7.02 \mathrm{e}-02$ \\
\hline Mt. Hopkins-CTIO/2MASS & $K_{\mathrm{s}}$ & 2.2 & $9.19 \mathrm{e}-03$ & $3.55 \mathrm{e}-04$ & $1.34 \mathrm{e}-02$ & $3.69 \mathrm{e}-04$ & 5.62 & $2.43 \mathrm{e}-01$ \\
\hline WISE & $W 1$ & 3.4 & $1.46 \mathrm{e}-02$ & $3.37 \mathrm{e}-04$ & $1.16 \mathrm{e}-02$ & $2.57 \mathrm{e}-04$ & 9.80 & $2.80 \mathrm{e}-01$ \\
\hline WISE & $W 2$ & 4.6 & $3.31 \mathrm{e}-02$ & $6.40 \mathrm{e}-04$ & $1.18 \mathrm{e}-02$ & $2.17 \mathrm{e}-04$ & 33.73 & $6.21 \mathrm{e}-02$ \\
\hline WISE & $W 3$ & 12 & $8.02 \mathrm{e}-02$ & $1.26 \mathrm{e}-03$ & $2.04 \mathrm{e}-03$ & $1.48 \mathrm{e}-04$ & 42.74 & $7.87 \mathrm{e}-02$ \\
\hline WISE & $W 4$ & 22 & $6.10 \mathrm{e}-01$ & $1.07 \mathrm{e}-02$ & $1.83 \mathrm{e}-03$ & $1.67 \mathrm{e}-01$ & 55.50 & $6.13 e-01$ \\
\hline Spitzer/IRAC & $I 1$ & 3.6 & $1.69 \mathrm{e}-02$ & $5.80 \mathrm{e}-05$ & $1.53 \mathrm{e}-02$ & $5.10 \mathrm{e}-05$ & $\ldots$ & $\ldots$ \\
\hline Spitzer/IRAC & $I 2$ & 4.5 & $2.48 \mathrm{e}-02$ & $4.40 \mathrm{e}-05$ & $1.11 \mathrm{e}-02$ & $3.40 \mathrm{e}-05$ & $\ldots$ & $\ldots$ \\
\hline Spitzer/IRAC & $\mathrm{I} 3$ & 5.8 & $3.44 \mathrm{e}-02$ & $1.17 \mathrm{e}-04$ & $1.02 \mathrm{e}-02$ & $5.10 \mathrm{e}-05$ & $\cdots$ & $\cdots$ \\
\hline Spitzer/IRAC & $I 4$ & 8.0 & $5.35 \mathrm{e}-02$ & $6.80 \mathrm{e}-05$ & $5.95 e-03$ & $2.00 \mathrm{e}-05$ & $\ldots$ & $\ldots$ \\
\hline Spitzer/MIPS & $M 1$ & 24 & $5.96 e-01$ & $2.95 \mathrm{e}-04$ & $6.87 \mathrm{e}-04$ & $8.90 \mathrm{e}-05$ & $\ldots$ & $\ldots$ \\
\hline Spitzer/MIPS & $M 2$ & 70 & 1.29 & $9.52 \mathrm{e}-03$ & $\ldots$ & $\ldots$ & $\ldots$ & $\ldots$ \\
\hline AKARI/IRC & $S 9 W$ & 9.2 & $\ldots$ & $\ldots$ & $\ldots$ & $\ldots$ & 30.07 & 1.90 \\
\hline AKARI/IRC & $L 18 W$ & 20 & $3.47 \mathrm{e}-01$ & $3.15 \mathrm{e}-02$ & $\ldots$ & $\ldots$ & 62.52 & 6.27 \\
\hline APEX/LABOCA & $\ldots$ & 870 & $1.09 \mathrm{e}-01$ & $5.00 \mathrm{e}-03$ & $\ldots$ & $\ldots$ & 1.75 & $4.00 \mathrm{e}-03$ \\
\hline
\end{tabular}

